the convergence abscissa (not necessarily positive) of a simple Dirichlet series.

Extensions of theorems of Vitali on sequences of analytic functions are given as Theorems VII and VIII. Theorem IX, concerning uniform convergence in a finite region and the consequent analyticity of the function represented, is essentially duplicated by Theorem 1, part 2, of $\mathrm{L}$, but is less general than Theorem 2 of AII on uniform convergence for an infinite region. Theorem $\mathrm{X}$ asserts that if the coefficients in (1) are all positive, $(S, T)$ is a singular place for the function represented. Theorem $\mathrm{XI}$, on simple Dirichlet series, is a preliminary to the proof of Theorem XII on the convergence of (1) in the region $R(s)=S$, $R(t)=T$.

Theorem XIII, on regular convergence of a double factorial series, is slightly less general than Theorem 1 of AI as supplemented by remarks on page 70 of $\mathrm{AI}$ and strengthened on page 407 of AII. Theorem XIV, on the identity of the related halfplanes of regular convergence for a factorial series and its associated special Dirichlet series, is duplicated by Theorem 3 of AII. Theorem $\mathrm{XV}$, on uniform convergence of a factorial series in a finite region, is slightly less general than the similar Theorem 2 of AI and much less general than Theorem 7 of AI (supplemented by footnote 12 of AII) on uniform convergence for an infinite region. Theorems XVI, XVII, and XVIII are respective analogs for binomial coefficient series of the preceding three theorems on factorial series.

BROWN UNIVERSITY

\title{
A CENSUS OF SQUARES OF ORDER 4, MAGIC IN ROWS, COLUMNS, AND DIAGONALS*
}

BY D. N. LEHMER

This census was made by Frenicle in 1693 who found 880 fundamental squares which with their rotations and reflections gave 7,040 squares of this sort. A study of the transformations which throw a square of this type into another of the same type shows that Frenicle need not have listed more than 220 fundamental squares. Besides his transformations, which were a rotation $R$ of order 4 of the square in its plane about a right angle,

* Presented to the Society, December 2, 1933. 
and a reflection $T$ of order 2 in a diagonal, there is a transformation $S$ of order 2 obtained by interchanging the 1st and 2d rows and the 1 st and $2 \mathrm{~d}$ columns. Also there is a transformation $U$ of order 2 obtained by interchanging the two middle rows and the two middle columns. These with the product transformation $U S$ serve to associate with any magic square of the sort in question 31 others so that the total number of squares should be a multiple of 32, and if Frenicle's result were correct one need list only 220 fundamental squares.

A set of fundamental squares must be such that no one of them can be obtained from any other by any of the transformations indicated above. The defining conditions were made to depend on the interesting fact that all of these transformations interchanged the corner elements among themselves, and the side elements among themselves. By a corner element is meant one that occupies a corner cell of the square or one of the four central cells. By a side element is meant any other. Attention was centered on the two largest entries 15 and 16 , and the squares were listed in four groups: (I) those in which 15 and 16 were corner elements; (II) those in which 16 was a corner element and 15 a side element; (III) those in which 15 was a corner element and 16 a side element; and (IV) those in which 15 and 16 were both side elements. Of the first set there were 31 fundamental squares; of the second there were 71 ; of the third there were also 71 and of the fourth there were 47 . The total $31+71+71+47=220$, and Frenicle's result is checked again, the total number being $220 \cdot 32=7,040$.

From this list of fundamental squares it is easy to pick out 6 which are magic also in the broken diagonals. It is interesting to note, however, that while the transformations $R, S$, and $T$ leave this property of "diabolism" unaltered, the transformations $U$ and $U S$ destroy it. The total number of such squares is therefore $6 \cdot 8=48$. The complete results for all squares of order 4 are then as follows:

Number of squares magic in rows and columns. .539,136*,

Number magic in rows, columns, and main diagonals. . .7,040, Number magic in rows, columns, and all diagonals......48.

The University of California

* This Bulletin, vol. 39 (1933), p. 767. 